\title{
Duplication and diversification of the LEAFY HULL STERILE1 and Oryza sativa MADS5 SEPALLATA lineages in graminoid Poales
}

\author{
Ashley R Christensen ${ }^{1,2}$ and Simon T Malcomber ${ }^{1 *}$
}

\begin{abstract}
Background: Gene duplication and the subsequent divergence in function of the resulting paralogs via subfunctionalization and/or neofunctionalization is hypothesized to have played a major role in the evolution of plant form. The LEAFY HULL STERILE1 (LHS1) SEPALLATA (SEP) genes have been linked with the origin and diversification of the grass spikelet, but it is uncertain 1) when the duplication event that produced the LHS1 clade and its paralogous lineage Oryza sativa MADS5 (OSM5) occurred, and 2) how changes in gene structure and/or expression might have contributed to subfunctionalization and/or neofunctionalization in the two lineages.

Methods: Phylogenetic relationships among 84 SEP genes were estimated using Bayesian methods. RNA expression patterns were inferred using in situ hybridization. The patterns of protein sequence and RNA expression evolution were reconstructed using maximum parsimony (MP) and maximum likelihood (ML) methods, respectively.

Results: Phylogenetic analyses mapped the LHS1/OSM5 duplication event to the base of the grass family. MP character reconstructions estimated a change from cytosine to thymine in the first codon position of the first amino acid after the Zea mays MADS3 (ZMM3) domain converted a glutamine to a stop codon in the OSM5 ancestor following the LHS1/OSM5 duplication event. RNA expression analyses of OSM5 co-orthologs in Avena sativa, Chasmanthium latifolium, Hordeum vulgare, Pennisetum glaucum, and Sorghum bicolor followed by ML reconstructions of these data and previously published analyses estimated a complex pattern of gain and loss of LHS1 and OSM5 expression in different floral organs and different flowers within the spikelet or inflorescence.

Conclusions: Previous authors have reported that rice OSM5 and LHS1 proteins have different interaction partners indicating that the truncation of OSM5 following the LHS1/OSM5 duplication event has resulted in both partitioned and potentially novel gene functions. The complex pattern of OSM5 and LHS1 expression evolution is not consistent with a simple subfunctionalization model following the gene duplication event, but there is evidence of recent partitioning of OSM5 and LHS1 expression within different floral organs of A. sativa, C. latifolium, P. glaucum and S. bicolor, and between the upper and lower florets of the two-flowered maize spikelet.
\end{abstract}

Keywords: grasses, LHS1, MADS-box, OsMADS5, Poaceae, neofunctionalization, subfunctionalization

\section{Background}

The diversification of paralogs following developmental gene duplication events is hypothesized to have played a major role in the evolution of morphological form [1-3]. Three general fates are hypothesized for duplicated gene

\footnotetext{
* Correspondence: simon.malcomber@csulb.edu

'Department of Biological Sciences, California State University - Long Beach,

1250 Bellflower Boulevard, Long Beach, CA 90840, USA

Full list of author information is available at the end of the article
}

products [2]. In the bulk of cases one of the gene products is predicted to accumulate mutations, become a pseudogene and be purged from the genome (pseudogenization). Alternatively, in a limited number of cases it is hypothesized that either changes to regulatory regions will result in the ancestral gene function being partially or completely partitioned between the two duplicates (subfunctionalization), or changes to regulatory domains and/or coding regions will result in a new

\section{Biomed Central}


gene function in one or both of the duplicates (neofunctionalization). Either the neofunctionalized developmental gene copies alone, or a combination of sub- and neofunctionalized copies are hypothesized to provide the raw genetic material necessary for the production of novel morphological structures.

The monocot clade comprises approximately 56,000 flowering plants, including such morphologically divergent lineages as orchids, palms, gingers, and grasses. Most monocot inflorescences contain one-many flowers, comprised of two whorls of three tepals, six stamens, and a three-carpellate pistil [4]. In contrast, grass inflorescences have, depending on the species, 1-40 flowers (or florets) collected into novel structures called grass spikelets [5]. At the base of each grass spikelet are two bractlike structures called glumes and each grass floret typically contains a lemma, a palea, two lodicules, three stamens, and a pistil. Sister to the grasses is a lineage comprised of the small families Joinvilleaceae and Ecdeiocoleaceae [6]. Joinvilleaceae and Ecdeiocoleaceae, in addition to other non-grass members of the graminoid Poales clade, have typical monocot flowers suggesting that a series of profound developmental and genetic changes occurred early in the history of the Poaceae lineage to produce grass spikelets, glumes, lemmas, paleas and lodicules typical of most taxa within the family.

The LOFSEP SEPALLATA (SEP) genes - containing the rice Oryza sativa MADS1/LEAFY HULL STERILE1 (OsMADS1/OsLHS1), Oryza sativa MADS5 (OSM5) and Oryza sativa MADS34/PANICLE PHYTOMER2 (OsMADS34/OsPAP2) genes, the petunia FLORAL BINDING PROTEIN9 (PhFBP9) and PhFBP23 genes, and Arabidopsis SEP1, SEP2 and SEP4 genes [7,8] - are hypothesized to have played a role in the origin and diversification of the grass spikelet $[8,9]$. SEP genes act as co-factors with $\mathrm{ABC}$ identity genes to help specify the identity of the different floral whorls [10,11]. Although the four Arabidopsis SEP (AtSEP1-4) genes are functionally redundant [12], at least two grass members of the LOFSEP clade, the rice OsLHS1/MADS1 and OsPAP2/MADS34 genes, have non-redundant roles during various stages of inflorescence development. Mutations to OsLHS1/MADS1 and OsPAP2/MADS34 are the basis for three named mutants: the OsLHS1/MADS1 mutants leafy hull sterile1 and naked seed rice (nsr), and the OsPAP2/MADS34 mutant panicle phytomer 2 (Ospap2). Both Oslhs1 and Osnsr are characterized by having leafy lemmas and paleas, leafy lodicules that resemble the lemma and palea, a decreased number of stamens, and occasionally, an extra pistil or floret $[13,14]$. Ospap2-1 mutants have more inflorescence branches, a disorganized arrangement of branches and spikelets, and elongated glumes and sterile lemmas compared to WT, but no obvious abnormalities in the lemma, palea, lodicules, stamens and pistil [15]. In a separate study, rice osmads34 mutants were reported as displaying an increase in the number of primary inflorescence branches, fewer secondary branches, fewer spikelets, and elongated sterile lemmas [16]. The difference in the number of secondary branches between the two studies is likely due to the different genetic backgrounds and type of mutations [16], but both studies agree on roles for the gene in regulating inflorescence branch number and sterile lemma morphology. Mutants of the remaining rice LOFSEP gene, osmads5, have only mild phenotypes compared to the WT plants, with lodicules attached to the palea and lemma [17]. Silencing of the rice $L H S 1$, OsMADS5 and the two SEP3 co-orthologs, OsMADS7 and OsMADS8 is sufficient to convert floral organs into leaf-like structures suggesting the four genes in concert perform the vast majority of SEP or E-class function during floral development [18].

Phylogenetic analyses of the monocot LOFSEP clade reconstruct the LHS1 and OSM5 clades as sister taxa, and the PAP2 clade as sister to a combined LHS1/ OSM5 clade $[3,19]$. These analyses suggest that both the LHS1/OSM5 and the LHS1+OSM5/PAP2 gene duplications occurred near the base of the grass family $[3,19]$, but additional sequences from early diverging grasses and other graminoid Poales are necessary to both test this hypothesis and provide a framework to investigate evidence of gene sub- and/or neofunctionalization within the lineage.

Expression patterns provide evidence of potential subfunctionalization and neofunctionalization between different paralogs [2]. Of the grass LOFSEP genes, expression patterns within the LHS1 clade are the most extensively studied and have been detected in the floral and spikelet meristems, in lemmas and paleas, and variously in the other floral organs, but not in glumes (except in wheat) $[9,20,21]$. Reinheimer et al. [20] used maximum parsimony (MP) character reconstructions and estimated an ancestral LHS1 expression pattern in the lemma, palea and carpels. LHS1 expression in lodicules was reconstructed to have been gained independently in the barley (Hordeum vulgare) lineage and at the base of the centothecoid and panicoid clade. LHS1 expression in stamens was gained independently in the maize (Zea mays) and Chasmanthium latifolium lineages, and LHS1 expression in the gynoecium was inferred to have been lost independently in the Eleusine indica and Paniceae (Pennisetum glaucum and Megathyrsus maximus) lineages. Based on this comparative analysis LHS1 was also hypothesized to act as a selector gene [22], specifying the terminal floret, in species whose spikelets mature basipetally, but not in species whose spikelets mature acropetally $[9,20]$. This complex pattern of LHS1 expression pattern in grasses led 
Malcomber and Kellogg [9] to hypothesize that differences in LHS1 expression pattern coupled with changes in interacting partner could have played a major role in the origin and diversification of the grass spikelet. Expression profiles of PAP2 and OSM5 co-orthologs in grasses are currently limited, but rice OsMADS34/PAP2 is broadly expressed in the floret meristem early in development, before becoming localized to the lemma, palea and stamens later in development [23]. Rice OSM5 is expressed in stamens and carpels, but not lemmas and paleas [24] and expression of the maize OSM5 co-ortholog, $Z m M 3$, is restricted to the lower floret [25].

Adding additional expression data from the OSM5 lineage and investigating the evolution of LOFSEP expression patterns within a phylogenetic context will not only provide new insights into possible evidence of sub- or neo-functionalization in the clades, but also provide insights into the patterns of gene evolution following duplication events.

\section{Methods}

\section{Plant Materials}

All plants were grown in greenhouses under natural light at 20 to $28^{\circ} \mathrm{C}$ with twice daily watering and regular fertilizing at California State University - Long Beach.

\section{Gene Isolation}

Total RNA was extracted from inflorescences, leaves and vegetative apices and cDNA synthesized as described by Woods et al. [26]. LOFSEP genes were isolated using either a semi-nested RACE PCR approach anchored using a polyT + adaptor primer (5'-CCG GAT CCT CTA GAG CGG CCG CTT TTT TTT TTT TTT TTT V-3') or using species specific primers with the reverse primer located in the 3' UTR (Additional File 1). All primers were designed using Primaclade [27]. PCR products were cleaned using silica spin column (Epoch Biolabs, Sugar Land, TX, US) and sub-cloned using a pGEM-T vector kit (Promega, Madison, WI, US). Both DNA strands were then sequenced using standard dideoxy sequencing protocols. The 35 new LOFSEP sequences isolated during this research have been deposited with the GenBank Data Libraries [Genbank: JN661596-JN661630]

\section{Expression Analysis}

RNA in situ hybridization of OSM5 co-orthologs in Avena sativa, C. latifolium, $H$. vulgare, P. glaucum and Sorghum bicolor were conducted on developing spikelets using 3' UTR probes derived from Reverse Transcription Polymerase Chain Reaction (RT-PCR) gene fragments as described by Malcomber and Kellogg [9]. Slides were imaged using an Olympus BX51 compound microscope with an Olympus DX4 digital camera. Hybridizations were repeated at least three times using different dissected and embedded materials to check for consistency. Images were cropped and adjusted for white balance using Adobe Photoshop CS5. Sense negative control hybridizations all showed no staining (data not shown).

\section{Phylogenetic and molecular evolutionary analyses}

LOFSEP genes were identified by Basic Local Alignment Search Tool (BLAST) searches at the National Center for Biotechnology Information (NCBI) (http://www.ncbi. nlm.nih.gov) and PlantGDB (http://www.plantgdb.org) using full-length coding regions of the rice OsLHS1/ MADS1 (Os03g11614), OsMADS5 (Os06g06750) and OsPAP2/MADS34 (Os03g54170) sequences as search seeds. Full-length sequences were assembled and translated into conceptual amino acids using Mesquite 2.74 [28] and then aligned using multiple sequence comparison by log-expectation (MUSCLE) [29] before being manually adjusted using MacClade 4.0 [30]. As in previous analyses of the $S E P$ gene family $[3,7,9,20]$, no regions were considered unalignable and excluded from subsequent analysis (See Additional File 2 for the phylogenetic data set). Bayesian phylogenetic analyses of the full-length LOFSEP data set using MrBayes 3.1.2 [31] were run on the Grethor parallel processing cluster at the University of Missouri - St. Louis and consisted of two separate searches of ten million generations using flat priors and the General Time Reversible (GTR) model of sequence evolution with invariant sites and gamma distributed rates partitioned according to codon position $(G T R+I+S S)$. Trees were sampled every 1,000 generations and convergence between the two runs was determined by examining the average standard deviation of the split frequencies. After ten million generations the split frequencies between the two runs was 0.006531 . After convergence had been assured the first $25 \%$ of trees were removed as burn-in and clade credibility values estimated using MrBayes.

The Shimodaira-Hasegawa test [32] for significance between the best maximum likelihood (ML) tree identified in the Bayesian search and the best tree consistent with the LHS1/OSM5 duplication mapping at the base of the combined Bambusoideae, Ehrhartoideae and Pooideae (BEP) and Panicoideae, Aristidoideae, Chloridoideae, Centothecoideae, Micrairoideae, Arundinoideae and Danthonioideae (PACCMAD) clade and after the divergence of Pharioideae was conducted in PAUP* 4.0 [33].

MP and ML character reconstruction analyses were conducted using Mesquite version 2.74 [28]. To facilitate comparisons among the different taxa we followed Whipple et al. [34], Sajo and Rudall [35] and Preston et al. [36] and considered: 1) bract 6 of the early diverging grass Streptochaeta angustifolia as homologous to one 
of the outer tepals in other monocots, a sepal in eudicots and the lemma in spikelet grasses, 2) bracts 7 and 8 of $S$. angustifolia as homologous to the remaining two outer tepals in other monocots, sepals in eudicots, and the palea of spikelet grasses, and 3) bracts 9-11 of $S$. angustifolia as homologous to the inner tepals in other monocots, petals in eudicots, and lodicules in spikelet grasses.

Tests for sites potentially under positive selection within the LOFSEP alignment utilized the best ML topology from the Bayesian search and the CODEML program within the Phylogenetic Analysis Using Maximum Likelihood (PAML) package [37] on the Grethor parallel processing cluster at University of Missouri - St Louis. Evidence of positive selection at particular codons was tested using the nested codon models M0 and M3, M1a and M2a, and M7 and M8 [38-40] with significance determined using a standard likelihood ratio test (LRT) statistic against a $\chi 2$ distribution with two degrees of freedom.

We also tested for sites potentially under positive selection on the branch subtending the LHS1 clade, the branch subtending the OSM5 clade, and the branch subtending the LHS1+OSM5 clades (Branches 1 to 3, Figure $1)$ using the modified branch-site models $A$ and $B$ $[39,40]$. Model A was compared with model M1a (NearlyNeutral) and model B was compared with M3 (discrete) with two site classes in a LRT against a $\chi 2$ distribution with two degrees of freedom $[39,40]$

\section{Results}

LOFSEP gene duplication and loss in graminoid Poales

Our Bayesian phylogenetic analysis of 84 LOFSEP genes estimated well-supported grass LHS1 (1.00 posterior probability [PP]), OSM5 (1.00 PP), and PAP2 (1.00 PP) clades (Figure 1 and Additional File 3 ). Although the sister relationship of the LHS1 and OSM5 subclades was only weakly supported (0.88 PP), the sister relationship of Joinvillea LHS1/OSM5-like (JaL1O5) to the LHS1/ OsMADS5 clade was well supported (1.00 PP). The first sequence to diverge within the LHS1 clade was isolated from a member of the earliest diverging lineage of grasses, S. angustifolia (subfamily Anomochlooideae), followed by a member of the earliest diverging grass lineage with true spikelets, Pharus latifolia (subfamily Pharioideae). To further investigate support for the estimated relationships we employed a Shimodaira-Hasegawa $(\mathrm{SH})$ test [32] to compare the best $\mathrm{ML}$ tree from the Bayesian search with the best ML tree satisfying the constraint that the LHS1/OSM5 duplication occurred at the base of the BEP+PACCMAD clades (after the divergence of $P$. latifolia). This analysis estimated the constraint tree (-ln 29886.59) as significantly less likely than the unconstrained analysis $(-\ln 23530.75, P<0.001)$.
Taken together, these data suggest that the LHS1/OSM5 (L1O5) duplication event occurred at the base of the grass family.

A clade of L1O5-like genes from two members of the graminoid Poales family Restionaceae was sister to JaL1O5, grass LHS1 and OSM5 clades, consistent with organismal relationships [6]. Both sampled members of the Restionaceae, Chondropetalum tectorum and Thamnochortus insignis,, had two L1O5 gene copies. C. tectorum L1O5a (CtL1O5a) and T. insignis L1O5a (TiL1O5a) formed a well-supported sister relationship (1.00 PP) that was sister to a CtL1O5b and TiL1O5b clade (1.00 PP), suggesting a gene duplication at the base of the African Restionaceae clade, or perhaps deeper within the lineage. A sequence isolated from a member of the Poales family Cyperaceae, Cyperus involucrata L1O5 (CiL1O5), was sister to the Restionaceae L1O5, JaL1O5, and grass LHS1/OsMADS5 clade (1.00 PP) suggesting an origin for the L1O5 LOFSEP clade at least within Poales, and potentially deeper within monocots.

Sister to the grass PAP2 clade was Joinvillea JaPAP2 (1.00 PP). This graminoid Poales clade was, in turn, sister to a clade of two oil palm sequences (Elias EgAGL24 and EgAGL2-5) that together comprised a well-supported commelinoid PAP2 clade (1.00 PP). Sister to the commelinoid PAP2 clade was a well-supported clade (0.99 PP) comprised of two orchid (Dendrobium DOMADS3 and Oncidium OgrMADS11) and two lily sequences (Lilium LIMADS3 and LIMADS4). The Poales L1O5 and monocot OsMADS34/PAP2 clades are, in turn, sister to an Acorus LOFSEP gene (AaAGL2, 1.00 $\mathrm{PP})$. Acorus is considered to be within the earliest diverging monocot lineage [4], suggesting that the gene duplication event to produce the PAP2 and L1O5 lineages occurred after the divergence of the Acorales, but prior to the divergence of the Liliales, Asparagales and Commelinoid clade.

\section{Molecular evolution of LOFSEP genes}

Changes in an amino acid sequence could have a deleterious, neutral or potentially beneficial effect on protein function by altering binding domains, changing protein stability, or modifying protein-folding ability. We used a combination of ML molecular evolutionary and MP character reconstruction approaches to investigate the changes in amino acid sequence prior to and following the LHS1/OSM5 duplication event at the base of the grass family. We first used the CODEML program within the PAML package [37] to test for evidence of positive selection using the nested models M0 and M3, M1a and M2a, M7 and M8 over the length of the aligned proteins followed by the branch-site models A and $\mathrm{B}$ on the branches subtending the LHS1, OSM5 and 


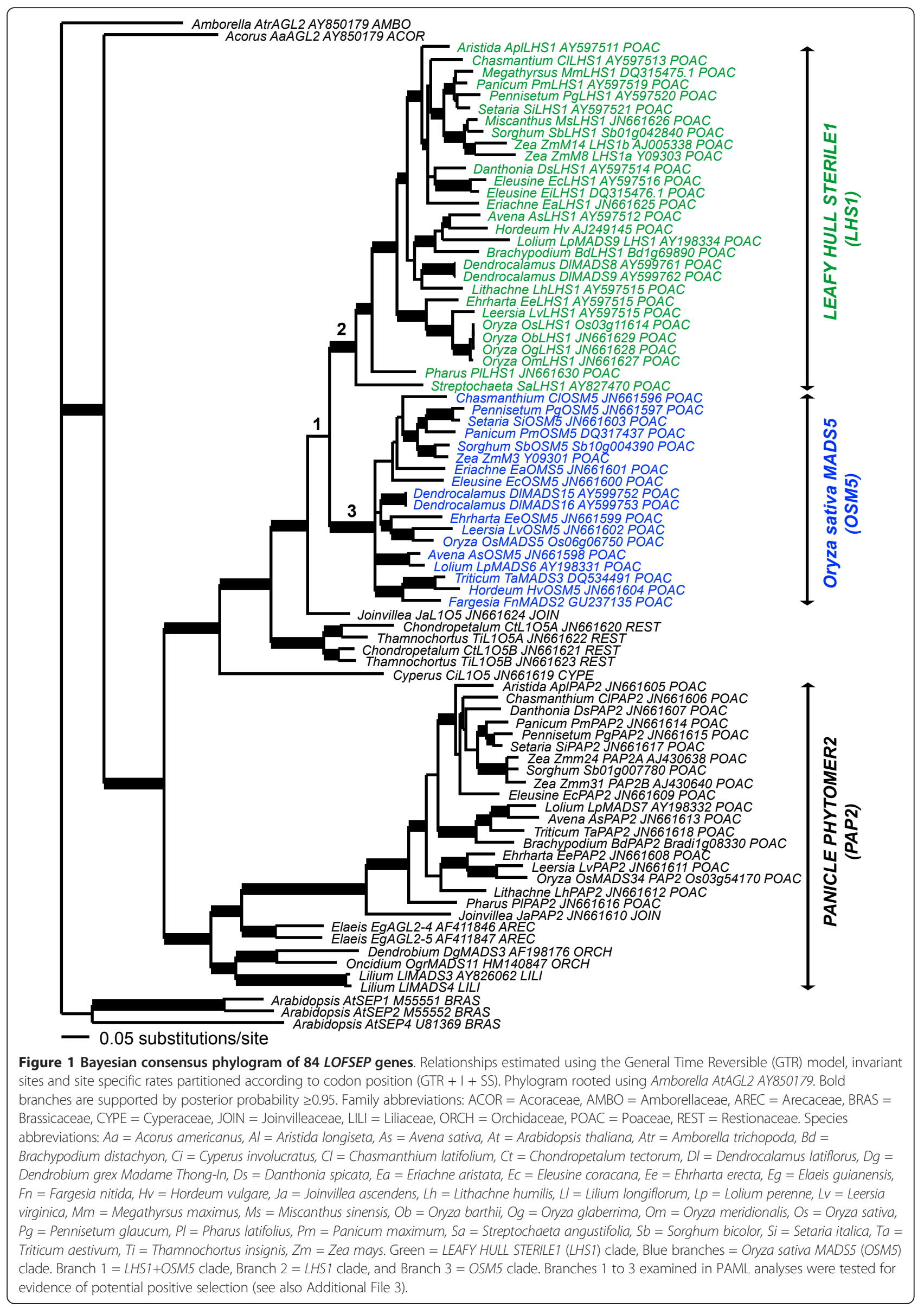


LHS1+OSM5 clades (Branches 1 to 3, Figure 1). All of these analyses recovered evidence of strong purifying selection (Additional File 4).

Of the four characteristic domains of MIKC-type MADS box genes, the C-terminal domain is both highly variable and critical for providing functional specificity via transcriptional activation [41]. Despite the lack of conservation of the C-terminal domain across the entire MADS-box gene family, individual gene clades often have conserved motifs [41]. Vandenbussche and colleagues [41] described two novel C-terminal motifs found in the monocot AGL2-like subfamily, ZMM3 and OSMADS1. We examined the sequence alignment of isolated LOFSEP-like proteins from members of the Restionaceae, Joinvilleaceae, and Poaceae to identify the presence or absence of the ZMM3 and OSMADS1 motifs. All sampled L1O5, LHS1, and OSM5 proteins in our analysis contained the ZMM3 motif. Both the L1O5 and LHS1 protein sequences also contained the
OSMADS1 motif, but this motif was lacking among OSM5 orthologs (Figure 2).

We then used MP character reconstruction methods to investigate nucleotide changes following the LHS1/ OSM5 duplication event that resulted in the truncation of OSM5 protein sequences. These analyses estimated a cytosine $(\mathrm{C})$ to thymine $(\mathrm{T})$ nucleotide substitution in the equivalent of the first codon position of the glutamine $(\mathrm{Q})$ in the $L 1 O 5$ common ancestor at the base of the OSM5 clade (Figure 3). This nucleotide substitution converted the ancestral glutamine into a stop codon in the first codon after the ZMM3 motif resulting in the truncation of OSM5 proteins (Figure 3). The MP nucleotide reconstruction analysis estimated ambiguity in the second codon position in the first codon of the ZMM3 domain with an adenine (A) or guanine (G) considered equally parsimonious. Neither of these changes, however, affected the reconstructed amino acid change from glutamine to a stop codon (Figure 3).

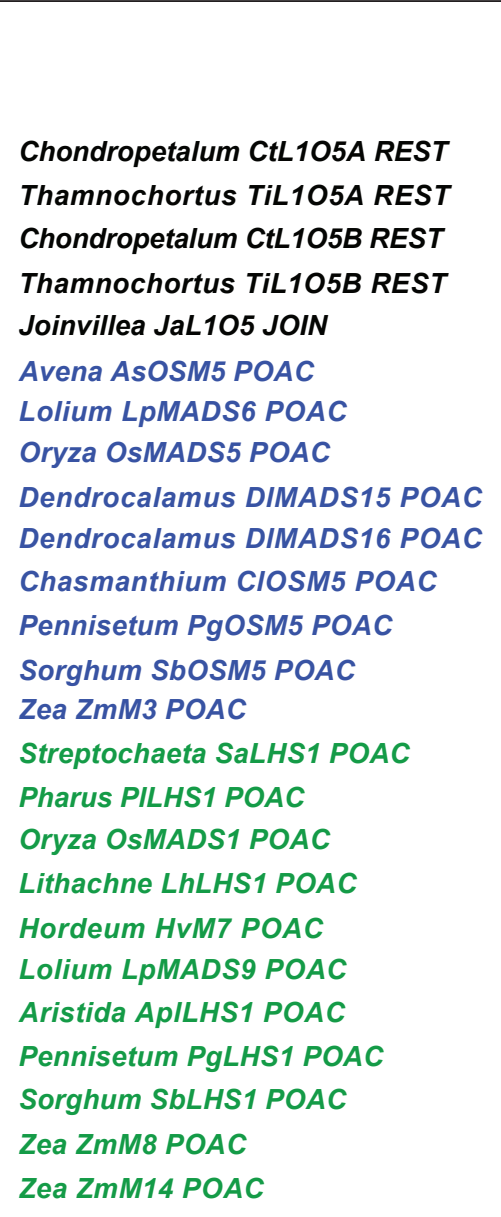

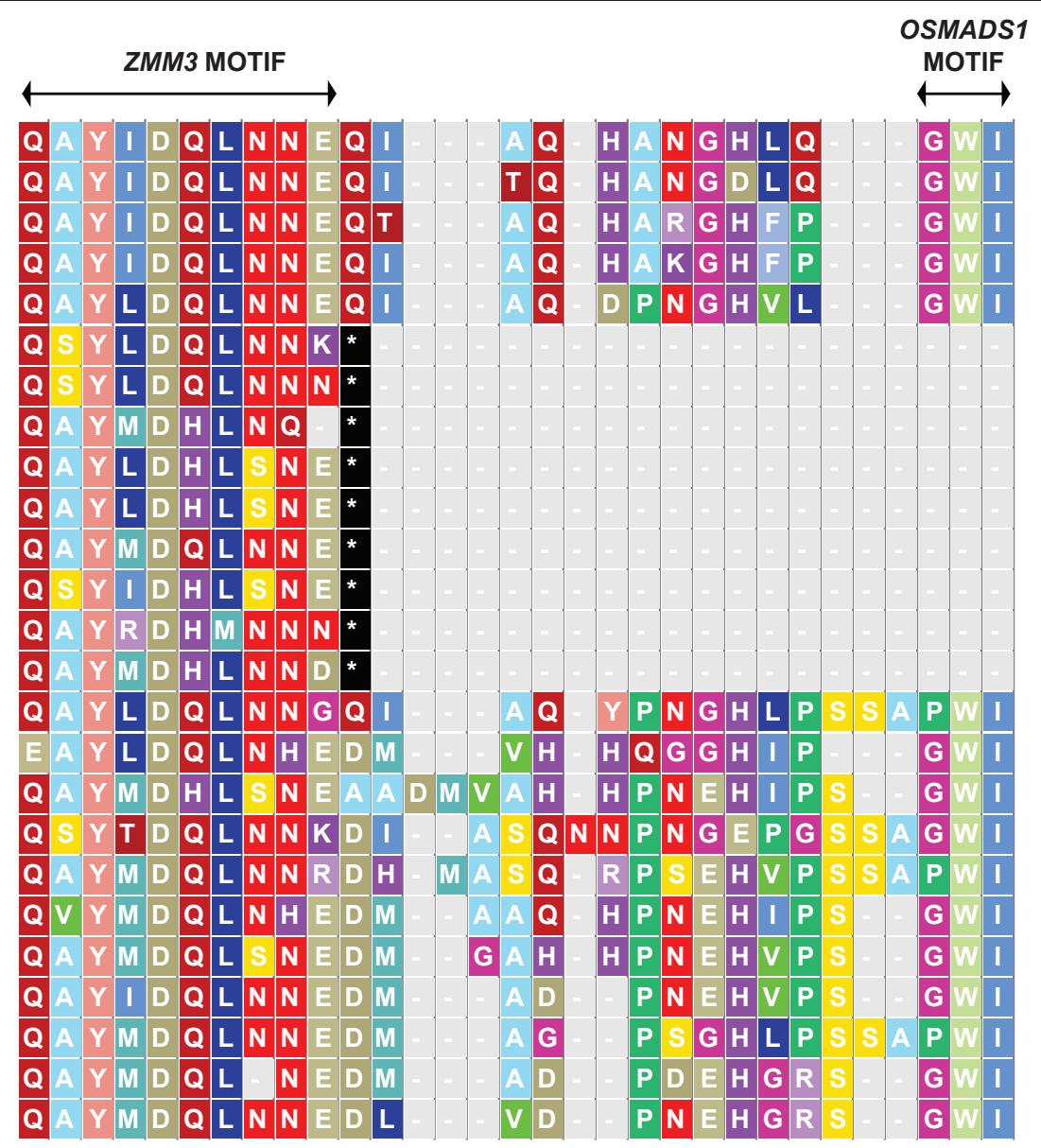

Figure 2 Alignment of amino acids within the C-terminus of graminoid Poales LOFSEP proteins. Proteins aligned using MUSCLE [29] and then manually adjusted. OSMADS1 and ZMM3 motifs identified by Vandenbussche et al.[41]. Green = LEAFY HULL STERILE1 (LHS1) clade, Blue branches = Oryza sativa MADS5 (OSM5) clade. 


\section{Heterogeneous OSM5 expression profiles}

Comparative mRNA expression studies in MADS box gene families are powerful tools to identify potential genetic interactors and infer whether expression patterns have diverged. To investigate the pattern of expression evolution within the OSM5 and LHS1 lineages we supplemented published LOFSEP gene expression profiles with mRNA expression data on OSM5 orthologs from a sample of taxa spanning the major diversification of the family.

A. sativa (subfamily Pooideae) has a two- to six-flowered, acropetally maturing spikelet (Figure 4A). A. sativa OSM5 (AsOSM5) was detected in lemmas and paleas within all florets of the developing spikelet (Figure 4B-D).

C. latifolium (subfamily Centothecoideae) has acropetally maturing spikelets comprised of 4 to 24 florets (Figure 4E). C. latifolium OSM5 (ClOSM5) was detected in the spikelet meristem and the developing palea, and in both the lemma and palea slightly later in development (Figure 4F-H).
H. vulgare (subfamily Pooideae) has an indeterminate inflorescence with one-flowered spikelets clustered together in triads on short secondary inflorescence branches (Figure 4I). Only the central spikelet is bisexual, whereas the two lateral spikelets are sterile and reduced to awns. $H$. vulgare OSM5 (HvOSM5) was detected within the lemma and lodicules in developing florets (Figure 4J-L).

P. glaucum (subfamily Panicoideae) has a two-flowered spikelet that matures basipetally. The upper floret is bisexual, whereas the lower floret is staminate or sterile. P. glaucum OSM5 (PgOSM5) was detected in the lodicules, stamens and carpels of the upper floret and the stamens and lodicules of the lower floret later in development (Figure 4M-N).

S. bicolor (subfamily Panicoideae) has an inflorescence comprised of sessile and pedicellate basipetally maturing two-flowered spikelets. The upper floret of the sessile spikelet is bisexual, whereas the upper floret of the pedicellate spikelet is staminate or sterile. The lower floret 


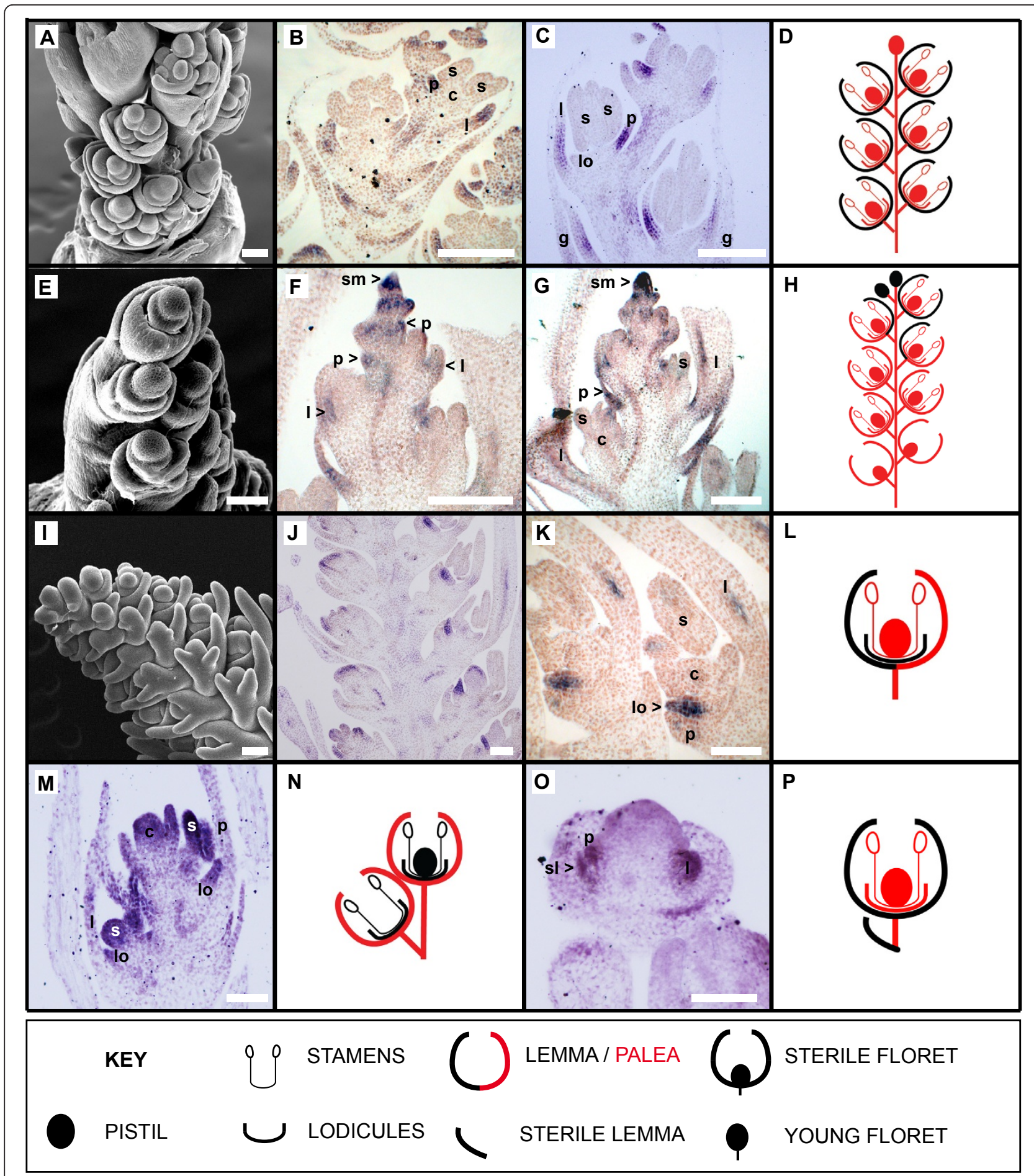

Figure 4 Scanning Electron Microscopy (SEM) analysis and mRNA expression profiles of OSM5 co-orthologs in Avena sativa, Chasmanthium latifolium, Hordeum vulgare, Pennisetum glaucum, and Sorghum bicolor inflorescences. Scanning electron microscopy

(SEM) images of inflorescence development in Avena sativa (A), Chasmanthium latifolium (E), and Hordeum vulgare (I), mRNA in situ hybridization expression analyses of OSM5 co-orthologs in developing inflorescences of A. sativa (B-C), C. latifolium (F-G), H. vulgare (J-K), P. glaucum (M) and S. bicolor (O) using antisense digoxygenin-labeled RNA probes, and cartoons summarizing OSM5 expression in the different taxa (D, H, L, N and P). Developing A. sativa spikelets with AsOSM5 expression in lemmas and paleas (B-D). Developing C. latifolium spikelets with CIOSM5 expression in the spikelet meristem, lemma and palea of upper florets and no expression in the lower florets (F-H). Developing $H$. vulgare spikelets with HvOSM5 expression in lemmas and lodicules (J-L). P. glaucum spikelets with PgOSM5 expression in carpels, stamens and lodicules of the upper floret and stamens and lodicules of the lower spikelet (M-N). Young S. bicolor spikelet with S6OSM5 expression in the palea and lemma of the upper floret and sterile lemma of the lower floret (O-P). Abbreviations: $c=$ carpels, I = lemma, lo = lodicule, $p=$ palea, $s=$ stamen, $s m=$ spikelet meristem. Bars, $100 \mu \mathrm{m}$. 
in both the sessile and pedicellate spikelets is reduced to a sterile lemma. S. bicolor OSM5 (SbOSM5) was detected in the lemma and palea of the upper floret and the sterile lemma of the lower floret (Figure 4O-P).

\section{Complex patterns of LHS1 and OSM5 mRNA expression evolution}

To investigate evidence of sub- or neofunctionalization following the L1O5 duplication event we combined our new expression data on OSM5 genes with published data on other LOFSEP genes [9,13,20-25,36,42-48] and reconstructed the pattern of evolution using ML within Mesquite.

All analyses recovered complex patterns of expression evolution in the different floral structures (Figure 5) and in different flowers/florets within the inflorescence (Figure 6). Joinvillea L1O5 is expressed within all floral whorls and all flowers within the inflorescence [36]. LHS1 was expressed in the lemma and palea of all sampled grasses, whereas OSM5 co-orthologs were also detected in the lemma and palea of A. sativa, C. latifolium, and S. bicolor, and the lemma of $H$. vulgare (Figure 5A). The presence of OSM5 expression within the lemma and palea was reconstructed as likely at the base of the clade, with the loss of expression estimated to have occurred independently in the rice and P. glaucum lineages.

Expression within whorl 2 is more homoplasious than whorl 1 (Figure 5B). LHS1 was detected in second whorl structures of $S$. angustifolia, $H$. vulgare, C. latifolium, $P$. glaucum and $Z$. mays whereas OSM5 was only expressed in lodicules of $H$. vulgare and P. glaucum (whether O. sativa OSM5 is expressed in lodicules is unknown). The reconstructed ancestral state at the base of the LHS1, OSM5 and LHS1+OSM5 clade is ambiguous in our analyses due to homoplasy within the clade, so it is uncertain whether expression was gained in those taxa expressing LHS1 or OSM5 in whorl 2 structures, or whether expression was lost in the other taxa.

The presence of expression in stamens was reconstructed as the more likely ancestral state at the base of the LHS1, OSM5 and LHS1+OSM5 clades (Figure 5C). LHS1 is expressed in the stamens of S. angustifolia, C. latifolium and Z. mays, and OSM5 is detected in the stamens of $O$. sativa and P. glaucum. ML character reconstructions estimate the independent loss of OSM5 expression in the pooid clade (A. sativa and $H$. vulgare) and at the base of PACCMAD grasses followed by the subsequent gain of expression in P. glaucum. LHS1 expression was lost on the branch subtending the BEP + PACCMAD clade and subsequently regained independently within the $C$. latifolium and $Z$. mays lineages.

The presence of expression within carpels was reconstructed as the ancestral state at the base of the LHS1 and LHS1+OSM5 clade, and marginally more likely at the base of the OSM5 clade (Figure 5D). OSM5 expression in carpels was subsequently lost in pooid grasses (A. sativa and $H$. vulgare) and at the base of panicoid +centothecoid clade, but subsequently regained within the P. glaucum lineage. Within the LHS1 clade, expression in carpels was independently lost in $H$. vulgare, $E$. indica, and in the Paniceae (M. maximum and $P$. glaucum).

Cacharrón and colleagues [22] hypothesized that the maize LHS1 and OSM5 co-orthologs. ZmM8, ZmM14 and $Z m M 3$, respectively, function as selector genes during inflorescence development. Expression of the maize LHS1 co-orthologs ZmM8 and ZmM14 were hypothesized to specify the terminal floret of the two-flowered spikelet [22], whereas expression of the OSM5 ortholog $\mathrm{ZmM} 3$ was hypothesized to specify the lower floret [43]. Using the expanded L1O5, LHS1 and OSM5 dataset we find no support for a subfunctionalization of LHS1 and OsMADS5 function into different flowers of the spikelet at the base of the two clades (Figure 6A). ML reconstructions estimate expression in all flowers at the base of the L1O5, LHS1 and OSM5 clades, with expression restricted to the upper flowers in O. sativa OSM5 and OsLHS1, M. maximus LHS1, P. glaucum LHS1, S. bicolor LHS1 and Z. mays ZmM8 and ZmM14. Expression restricted to the lower floret is only reported in maize $Z m M 3$, and based on our reconstructions this partitioning of expression evolved relatively recently (since maize and sorghum last shared a common ancestor).

Together, these data do not support the subfunctionalization of LHS1 and OSM5 function into different regions within the grass spikelet following the L1O5 duplication event. However, our analyses are consistent with LHS1 functioning as a selector gene of the terminal flower in basipetally maturing spikelets of ehrhartoid and panicoid grasses.

\section{Discussion}

LHS1 genes have been linked to the origin and diversification of the grass spikelet, but the role of its paralog, OSM5, has largely been overlooked. In this analysis we have used a combination of Bayesian phylogenetics, expression analyses, molecular evolutionary analyses, and MP and ML character reconstruction methods to investigate the evolutionary history of the LHS1 and OSM5 lineages of SEP genes. These analyses reconstruct a complex pattern of duplication and diversification and provide new insights into how these genes are expressed during spikelet and floret development in grasses.

\section{The LHS1/OSM5 duplication event maps to the base of the grass family}

Our Bayesian phylogenetic analyses support the hypothesis that the LHS1 and OSM5 lineages are products of a 


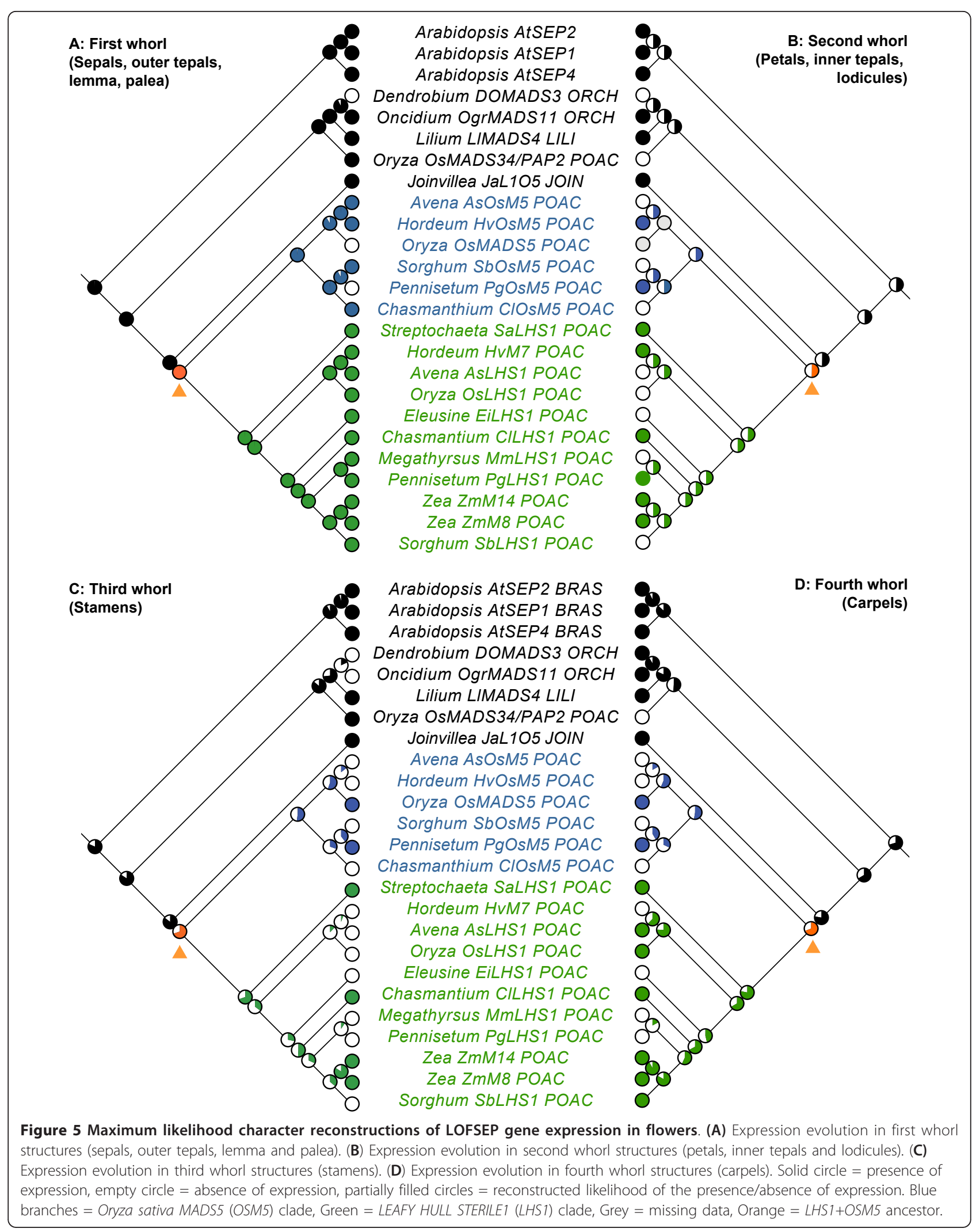




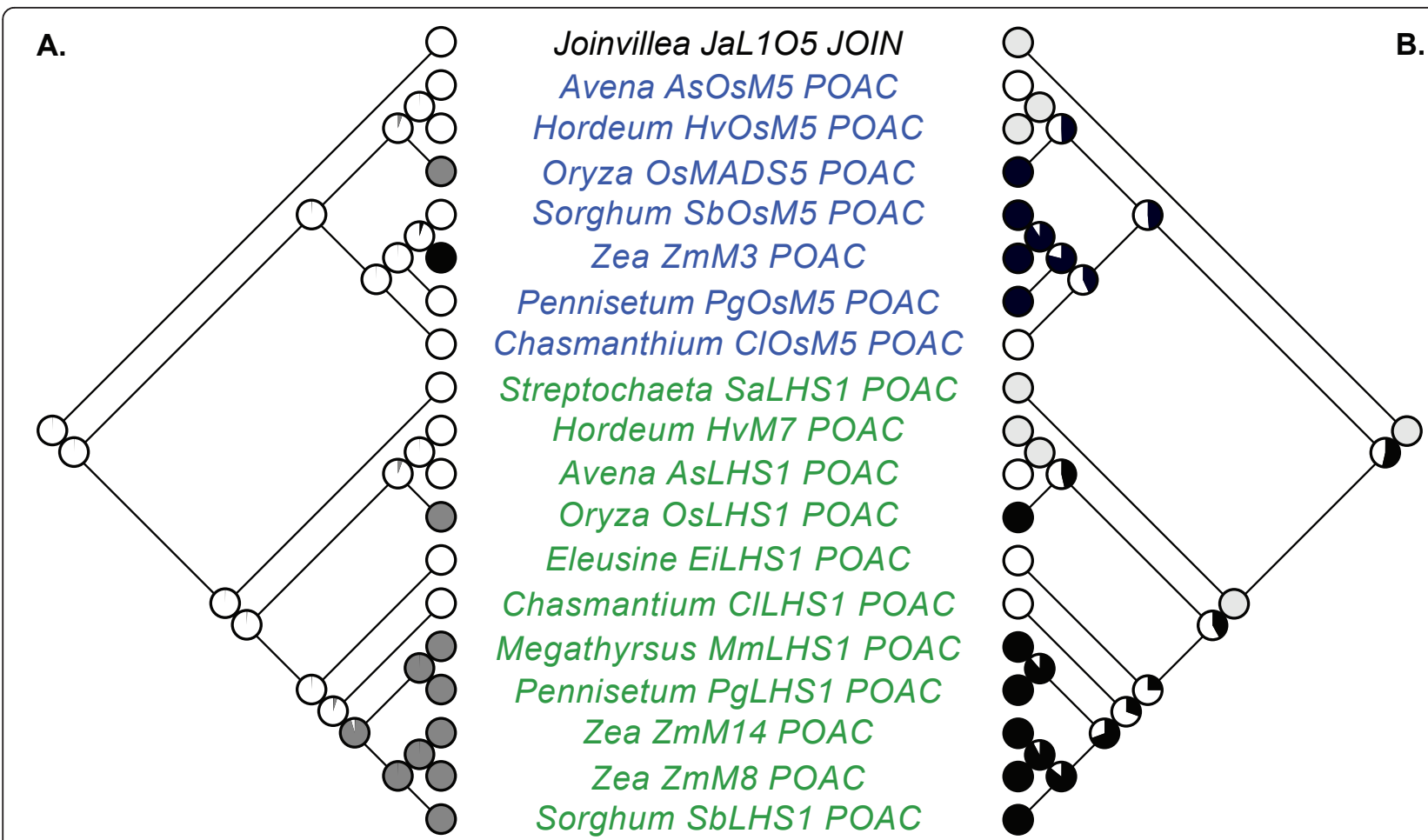

Figure 6 Maximum likelihood character reconstructions of graminoid poales LOFSEP gene expression in inflorescences and spikelets. (A) Expression evolution in different regions of the inflorescence and spikelet. Black = Expression restricted to the lower floret within the spikelet, Grey $=$ Expression restricted to the upper floret in the spikelet, White $=$ Expression present in all flowers of the inflorescence. (B) Evolution of floral maturation. Black = basipetally maturing spikelets, Grey = Flowers borne singly or spikelets comprised of a single floret, White = acropetally maturing spikelets. Green = LEAFY HULL STERILE1 (LHS1) clade, Blue branches = Oryza sativa MADS5 (OSM5) clade.

gene duplication event at the base of the grass family. Although LHS1 co-orthologs were isolated from $S$. angustifolia and P. latifolia, the two earliest diverging members of the Poaceae, we were unable to isolate OSM5 co-orthologs from these taxa. The most parsimonious explanation hypothesizes independent losses of the OsMS5 co-orthologs in the two lineages. However, it is also possible that these OSM5 genes: 1) were expressed at a developmental stage not represented in our cDNA, 2) are too divergent to amplify using our degenerate primers, or 3) that the Bayesian tree has reconstructed the position of the LHS1/OSM5 duplication event incorrectly. We consider the independent loss hypothesis more likely based on three lines of evidence. First, the cDNA stocks used to isolate the genes using RT-PCR spanned diverse stages of inflorescence development, ranging from early branching through to floral organ initiation and maturation. Second, we were able to successfully amplify LOFSEP (Figure 1), SEP3 (Christensen and Malcomber, unpublished) and AGL6-like genes [49] from diverse other Poales using the same degenerate MADS primers and cDNA stocks. Third, the results of a Shimodaira-Hasegawa test [32] indicated that a constrained phylogeny where the LHS1/OSM5 duplication event occurred at the base of the BEP and PACCMAD clade and after the divergence of the Anomochlooideae and Pharioideae was significantly less likely $(P<0.001)$ than the unconstrained analysis with a duplication event at the base of the grass family.

A similar pattern of gene loss in S. angustifolia and $P$. latifolia was also reported in FRUITFULL (FUL) genes. In this analysis, Preston and Kellogg [50] were able to isolate FUL2 co-orthologs from S. angustifolia and $P$. latifolia, but not FUL1. Rice OsFUL1 and OsLHS1 both occur on chomosome 3, whereas OsFUL2 and OsMADS5 both occur on chromosome 6. Because OSM5 and OSFUL1 occur on different chromosomes we hypothesize that a more localized pattern of independent gene loss in the Anomochlooideae and Pharioideae produced the complex pattern of relationships within FUL and LOFSEP lineages rather than a pair of large chromosomal deletion events.

\section{OSM5 proteins are truncated relative to LHS1 and L1O5 proteins}

Our molecular evolutionary analyses of the LHS1, OSM5 and $L 105$ clade failed to find any evidence of positive selection (Additional file 4), but MP reconstructions 
estimated a cytosine to thymine substitution in the first codon position of the first amino acid immediately after the $Z M M 3$ domain that converted a glutamine into stop codon and truncated OSM5 relative to the ancestral L1O5 proteins. This is a different hypothesis than proposed by $\mathrm{Xu}$ and Kong [19] who hypothesized that the insertion of a cytosine within the exon 8 of the rice OsM5 gene resulted in a frameshift and introduction of a premature stop codon. Our phylogenetic reconstructions of the LOFSEP data set estimate the insertion-deletion event within the rice OSM5 ZMM3 domain occurred relatively recently within the evolutionary history of the grass family (since ehrhartoid and pooid grasses last shared a common ancestor) and consequently did not coincide with the duplication event giving rise to the OSM5 lineage at the base of the grass family.

The C-terminal regions of MADS box genes are known to help regulate transcriptional activation, partner specificity, subcellular localization and/or the ability to attract interacting partners $[8,41]$. Deletion of amino acids 218 to 257 of the rice LHS1 protein, including both the ZMM3 and OSMADS1 domains, removed the ability of the protein to bind FUL1/OsMADS14 and FUL2/OsMADS15 [51] confirming a role for the C-terminus in regulating partner interactions. Cui et al. [18] reported that rice LHS1 and OSM5 proteins interact differently; OsLHS1 can homodimerize weakly and heterodimerize with the AGL11-like protein OsMADS13 and the SEP3 proteins OsMADS7 and OsMADS8, whereas rice OSM5 cannot homodimerize and cannot heterodimerize with OsLHS1, OsMADS7, OsMADS8 or OsMADS13. Whether rice LHS1 and OSM5 can heterodimerize with PAP2 has yet to be shown, but the discrepancy in binding abilities between OsLHS1 and OSM5 suggests that the truncation within the $\mathrm{C}$-terminus of the OSM5 protein following the duplication event at the base of the grass family has had a profound effect on its ability to form multimeric complexes. The subtle phenotypic differences in rice osmads 5 mutants with lodicules attached to the lemma and palea [17] suggests that OSM5 has a partially redundant role with other E-class genes during floral development. However, the inability of OSM5 to form complexes with the same set of proteins as OsLHS1 indicates that OSM5 is not (partially) redundant with OsLHS1. Together these data suggest that changes following the LHS1/OSM5 duplication have resulted in a new coding region function (or at least the loss of several functions currently performed by LHS1) for the OSM5 lineage and potentially some partitioning of function between LHS1 and OsMS lineages.

Complex patterns of expression of OSM5 and LHS1 genes Our analysis of OSM 5 and LHS1 mRNA expression profiles reveals a complex pattern of complimentary, overlapping and absent expression profiles within flowers (Figure 5) and in different regions of the inflorescence (Figure 6). Complimentary mRNA expression profiles of OSM5 and LHS1 occur in maize, where $Z m M 3$ is restricted to the lower floret while $Z m M 8$ and $Z m M 14$ are only expressed in the upper floret, and within floral organs of certain sampled taxa, including: 1) the lemmas of rice and P. glaucum where LHS1 is expressed, but OSM5 is not, 2) the lodicules of C. latifolium where LHS1 is expressed, but OSM5 is not, 3) the stamens of rice and P. glaucum where OSM5 is expressed, but LHS1 is not, and 4) the carpels of $A$. sativa, C. latifolium and S. bicolor where LHS1 is expressed, but OSM5 is not, and in P. glaucum where OSM5 is expressed, but LHS1 is not. Several species also show overlapping expression profiles with LHS1 and OSM 5 both being expressed in the lemmas and paleas of A. sativa, S. bicolor and C. latifolium, in the lemmas of $H$. vulgare, in the lodicules of $H$. vulgare and $P$. glaucum, and in the carpels of rice. Neither LHS1 nor OSM5 are expressed in the lodicules of S. bicolor, the stamens of $A$. sativa and S. bicolor, and the stamens and carpels of $H$. vulgare suggesting the E-class functional role in these structures is provided by either PAP2 or, more likely based on studies in rice [18], SEP3 co-orthologs. Taken together these data suggest a more labile pattern of expression evolution within the LHS1 and OSM5 clades than predicted by the classic model of subfunctionalization [1].

Expression profiles of LHS1, OSM5, PAP2 and the SEP3 co-orthologs OSM7 and OSM8 in rice osmads7, osmads 8 , osmads $7 / 8$ and osmads $1 / 5 / 7 / 8$ mutants provide additional insights into the plasticity of $S E P$ gene expression in grasses and their roles during floral development. In osmads7 mutants, LHS1 (approximately 1.3 to $1.5 \times$ WT expression), OSM5 (approximately 2.5 to $3 \times$ WT expression), OSM8 (approximately 1.3 to $1.7 \times \mathrm{WT}$ expression) and PAP2 (approximately 1.2 to $1.3 \times \mathrm{WT}$ expression) were upregulated to compensate for the lack of OSM7 expression and in osmads8 mutants LHS1 (approximately 1.5 to $1.7 \times$ WT expression), OSM5 (approximately 1.8 to $2.1 \times$ WT expression), OSM7 (approximately 2.2 to $2.4 \times \mathrm{WT}$ expression) and PAP2 (approximately 1.3 to $1.5 \times$ WT expression) were all upregulated and sufficient to produce mutant plants with only subtle phenotypes [18]. However, in osmads7/ 8 mutants even the upregulation of LHS1 (approximately 2.5 to $3.6 \times$ WT expression), OSM5 (approximately 1.6 to $2 \times$ WT expression), and PAP2 (approximately 1.7 to $1.8 \times$ WT expression) still produced severe mutant plants with two to four lodicules transformed into lemma and palea-like structures and three to seven longer and thinner sterile stamens [18], suggesting that the rice LOFSEP (LHS1, OSM5 and 
$P A P 2)$ genes are functionally divergent from the SEP3 co-orthologs and unable to complement the mutant. In osmads1/5/7/8 mutants, PAP2 expression ranged from 1.2 to $1.6 \times$ WT expression levels but even this increased expression was, again, unable to compensate for the lack of the other SEP genes with mutant plants having flowering delayed by three to four weeks, shorter inflorescences with increased branching, and florets with leaflike lemma, palea, lodicules, stamens and carpels [18]. Given the inability of LHS1, OSM5 and PAP2 to compensate for the lack of OSM7 and OSM8 in the mutant and the inability of PAP2 to compensate for the lack of SEP expression in the quadruple osmads1/5/7/8 mutant, these data point to either a direct or indirect negative feedback loop regulating expression among the different rice SEP genes, rather than a dosage effect among partially redundant genes [18].

This feedback loop could have compensated for the lack of OsMADS5 in rice osmads 5 mutants which in turn resulted in plants with only subtle phenotypes. The different protein binding abilities of rice LHS1 and OSM5 suggests one (or more) of the other of the rice SEP genes is partially compensating for OsMADS5 in these mutants. Which of these other SEP genes, however, awaits further analysis of rice osmads 5 mutants using real time RT-PCR.

\section{Conclusions}

The grass LOFSEP genes LHS1, OSM5 and PAP2 all regulate aspects of grass inflorescence development, but the timing of the duplication events to produce the different lineages and the pattern of evolution within the different clades has not been fully investigated. In this analysis we used Bayesian phylogenetic methods to reconstruct relationships among 84 LOFSEP SEPALLATA genes that map the LHS1 and OSM5 duplication event at the very base of the grass family, whereas the LHS1+OSM5 and PAP2 duplication event maps to deeper within the monocot clade. MP reconstructions within the LHS1 and OSM5 lineage estimated a cytosine to thymine substitution converted a glutamine to stop codon immediately after the ZMM3 domain that truncated OSM5 relative to LHS1 and the $\mathrm{L} 1 \mathrm{O} 5$ ancestor. Based on studies in rice, the truncation of the OSM5 protein and removal of the OSMADS1 domain has resulted in a different set of protein interaction partners [18,51]. This observation supports hypotheses of both subfunctionalization between LHS1 and OSM5 and neofunctionalization within the OSM5 lineage via a change in the coding region. ML character reconstruction analyses estimate a complex pattern of OSM 5 and LHS1 expression evolution that is not consistent with a classic subfunctionalization model of partitioning following the gene duplication event. However, our analyses do support a hypothesis of recent OSM5 and
LHS1 expression partitioning within the floral organs of $A$. sativa, C. latifolium, H. vulgare, P. glaucum and S. bicolor, and between the upper and lower florets of the two-flowered maize spikelet. How this complex pattern of gene expression evolution within the two lineages has affected morphological evolution in grasses has yet to be determined. The next step will be to expand expression analyses within the OSM5 lineage and couple these studies with investigations of OSM 5 and LHS1 protein interactions and functional analyses of osmads 5 and $l$ hs 1 single and double mutants in transformable grasses such as Brachypodium distachyon [52-54] and Setaria viridis [55].

\section{Additional material}

Additional file 1: Oligonucleotide primer combinations and primer sequences used to amplify LOFSEP genes from graminoid Poales. Additional file 2: Aligned LOFSEP Bayesian data set.

Additional file 3: LOFSEP phylogenetic tree with posterior probabilities.

Additional file 4: Summary of LOFSEP molecular evolutionary analyses using the CODEML package within PAML.

\section{Abbreviations}

AsOSM5: Avena sativa ORYZA SATIVA MADS5; BEP: Bambusoideae, Ehrhartoideae and Pooideae; BLAST: Basic Local Alignment Search Tool; CIOSM5: Chasmanthium latifolium ORYZA SATIVA MADS5; FUL, FRUITFULL; HVOSM5: Hordeum vulgare ORYZA SATIVA MADS5; L1O5: LEAFY HULL STERILE1/ Oryza sativa MADS5-like; LHS1: LEAFY HULL STERILE1; LOFSEP: Oryza sativa MADS1/LEAFY HULL STERILE1, Oryza sativa MADS5, Oryza sativa MADS34/ PANICLE'PHYTOMER2, petunia FLORAL BINDING PROTEIN9 (PhFBP9) and PhFBP23 genes, and Arabidopsis SEP1, SEP2 and SEP4; ML: Maximum Likelihood; MP: Maximum Parsimony; NCBI: National Center for Biotechnology Information; nsr: naked seed rice; OsLHS1: Oryza sativa LEAFY HULL STERILE1; OSMADS1: Oryza sativa MADS1; OSMADS34: Oryza sativa MADS34; OSM5: Oryza sativa MADS5; OsMADS7: Oryza sativa MADS7; OsMADS8: Oryza sativa MADS8; OsPAP2: Oryza sativa PANICLE PHYTOMER2; PACCMAD: Panicoideae, Aristidoideae, Chloridoideae, Centothecoideae, Micrairoideae, Arundinoideae and Danthonioideae; PAML: Phylogenetic Analysis Using Maximum Likelihood; PgOSM5: Pennisetum glaucum ORYZA SATIVA MADS5; PhFBP9: Petunia hybrida FLORAL BINDING PROTEIN9; PhFBP23: Petunia hybrida FLORAL BINDING PROTEIN23; PP: Posterior Probability; RACE PCR: Randomly Amplified cDNA Ends Polymerase Chain Reaction; RT-PCR: Reverse Transcription Polymerase Chain Reaction; SEP: SEPALLATA; SH: Shimodaira-Hasegawa; SbOSM5: Sorghum bicolor ORYZA SATIVA MADS5; UTR: Untranslated Region; ZMM3: Zea mays MADS3...\}

\section{Acknowledgements}

This research was supported by NSF DEB-0716788 (SM). Bayesian phylogenetic analyses and tests for positive selection using the CODEML package within PAML were conducted on the Grethor parallel processing cluster at the University of Missouri - St. Louis. SEM analyses were conducted at the Institute for Integrated Research on Materials, Environment and Society (IIRMES) at CSULB. We would also like to thank two anonymous reviewers for comments and David Lorence, Renata Reinheimer and Elizabeth Kellogg for plant materials.

\section{Author details}

'Department of Biological Sciences, California State University - Long Beach, 1250 Bellflower Boulevard, Long Beach, CA 90840, USA. ${ }^{2}$ Agensys, Inc., an Affiliate of Astellas Pharma Inc., $154517^{\text {th }}$ Street, Santa Monica, CA 90404, USA. 


\section{Authors' contributions}

AC isolated and aligned LOFSEP genes from non-model graminoid Poales, conducted in situ hybridization expression and PAML analyses, examined morphological development using SEM, and drafted the manuscript. SM conceived the study, conducted in situ hybridization expression, phylogenetic and character reconstruction analyses, and helped draft the manuscript. Both authors read and approved the final manuscript.

\section{Competing interests}

The authors declare that they have no competing interests.

Received: 18 November 2011 Accepted: 17 February 2012

Published: 17 February 2012

\section{References}

1. Force A, Lynch M, Pickett FB, Amores A, Yan YL, Postlethwait J: Preservation of duplicate genes by complementary, degenerative mutations. Genetics 1999, 151:1531-1545.

2. Moore RC, Purugganan MD: The evolutionary dynamics of plant duplicate genes. Curr Opin Plant Biol 2005, 8:122-128.

3. Malcomber ST, Preston JC, Reinheimer R, Kossuth J, Kellogg EA: Developmental gene evolution and the origin of grass inflorescence diversity. In Advances in Botanical Research. Volume 44. Edited by: Soltis DE. JHLPSS, Callow JA: Academic Press; 2006:425-481.

4. Angiosperm Phylogeny Website., Version 9, June 2008 [and more or less continuously updated since], [http://www.mobot.org/MOBOT/research/ APweb/].

5. Clifford HT: Spikelet and floral morphology. In Grass Systematics and Evolution. Edited by: Barkworth ME. Washington, DC: Smithsonian Institute; 1987:21-30.

6. Marchant AD, Briggs BG: Ecdeiocoleaceae and Joinvilleaceae, sisters of Poaceae (Poales): evidence from rbcL and matK data. Telopea 2007, 11:437-450.

7. Zahn LM, Kong H, Leebens-Mack JH, Kim S, Soltis PS, Landherr LL, Soltis DE, Depamphilis CW, Ma H: The evolution of the SEPALLATA subfamily of MADS-box genes: a pre-angiosperm origin with multiple duplications throughout angiosperm history. Genetics 2005, 169:2225-2239.

8. Malcomber ST, Kellogg EA: SEPALLATA gene diversification: brave new whorls. Trends Plant Sci 2005, 10:427-435.

9. Malcomber ST, Kellogg EA: Heterogeneous expression patterns and separate roles of the SEPALLATA gene LEAFY HULL STERILE1 in grasses. Plant Cell 2004, 16:1692-1706

10. Theissen G, Saedler H: Plant biology. Floral quartets. Nature 2001, 409:469-471

11. Honma T, Goto K: Complexes of MADS-box proteins are sufficient to convert leaves into floral organs. Nature 2001, 409:525-529.

12. Pelaz S, Ditta GS, Baumann E, Wisman E, Yanofsky MF: B and C floral organ identity functions require SEPALLATA MADS-box genes. Nature 2000, 405:200-203.

13. Jeon JS, Jang $S$, Lee $S$, Nam J, Kim C, Lee SH, Chung YY, Kim SR, Lee YH, Cho YG, An G: leafy hull sterile1 is a homeotic mutation in a rice MADS box gene affecting rice flower development. Plant Cell 2000, 12:871-884

14. Chen ZX, Wu JG, Ding WN, Chen HM, Wu P, Shi CH: Morphogenesis and molecular basis on naked seed rice, a novel homeotic mutation of OsMADS1 regulating transcript level of AP3 homologue in rice. Planta 2006, 223:882-890

15. Kobayashi K, Maekawa M, Miyao A, Hirochika H, Kyozuka J: PANICLE PHYTOMER2 (PAP2), encoding a SEPALLATA subfamily MADS-box protein, positively controls spikelet meristem identity in rice. Plant Cell Physiol 2010, 51:47-57

16. Gao X, Liang W, Yin C, Ji S, Wang H, Su X, Guo C, Kong H, Xue H, Zhang D: The SEPALLATA-like gene OSMADS34 is required for rice inflorescence and spikelet development. Plant Physiol 2010, 153:728-740.

17. Agrawal G, Abe K, Yamazaki M, Miyao A, Hirochika H: Conservation of the E-function for floral organ Identity in rice revealed by the analysis of tissue culture-induced loss-of-function mutants of the OsMADS1 Gene. Plant Mol Biol 2005, 59:125-135.

18. Cui R, Han J, Zhao S, Su K, Wu F, Du X, Xu Q, Chong K, Theissen G, Meng Z: Functional conservation and diversification of class $\mathrm{E}$ floral homeotic genes in rice (Oryza sativa). Plant J 2010, 61:767-781.
19. Xu G, Kong H: Duplication and divergence of floral MADS-Box genes in grasses: Evidence for the generation and modification of novel regulators. J Integ Plant Biol 2007, 49:927-939.

20. Reinheimer R, Malcomber $S$, Kellogg EA: Evidence for distinct roles of the SEPALLATA genes LEAFY HULL STERILE1 in Eleusine indica and Megathyrsus maximus (Poaceae). Evol Dev 2006, 8:293-303.

21. Shitsukawa N, Tahira C, Kassai K-i, Hirabayashi C, Shimizu T, Takumi S, Mochida K, Kawaura K, Ogihara Y, Murai K: Genetic and epigenetic alteration among three homoeologous genes of a Class E MADS-box gene in hexaploid wheat. Plant Cell 2007, 19:1723-1737.

22. Cacharrón J, Saedler $H$, Theissen G: Expression of MADS box genes ZMM8 and ZMM14 during inflorescence development of Zea mays discriminates between the upper and the lower floret of each spikelet. Dev Genes Evol 1999, 209:411-420.

23. Pelucchi N, Fornara F, Favalli C, Masiero S, Lago C, Pe ME, Colombo L, Kater MM: Comparative analysis of rice MADS-box genes expressed during flower development. Sex Plant Reprod 2002, 15:113-122.

24. Kang $H G$, An GH: Isolation and characterization of a rice MADS box gene belonging to the AGL2 gene family. Mol Cells 1997, 7:45-51.

25. Deleu WKP: Molecular and Functional Analysis of AGL2-like MADS-box genes in Maize (Zea mays ssp. mays): Indications for their involvement in grass inflorescence architecture. PhD Thesis Universität zu Köln, Mathematisch-Naturwissenschaftlichen Fakultät; 2002.

26. Woods DP, Hope CL, Malcomber ST: Phylogenomic analyses of the BARREN STALK1/LAX PANICLE1 (BA1/LAX1) genes and evidence for their roles during axillary meristem development. Mol Biol Evol 2011, 28:2147-2159.

27. Gadberry MD, Malcomber ST, Doust AN, Kellogg EA: Primaclade-a flexible tool to find conserved PCR primers across multiple species. Bioinformatics 2005, 21:1263-1264.

28. Maddison WP, Maddison DR: Mesquite: a modular system for evolutionary analysis., Version 2.74 [http://www.mesquiteproject.org].

29. Edgar RC: MUSCLE: a multiple sequence alignment method with reduced time and space complexity. BMC Bioinformatics 2004, 5:113.

30. Maddison DR, Maddison WP: MacClade: Analysis of phylogeny and character evolution. 2003, Version 4.06. Sinauer Associates.

31. Ronquist F, Huelsenbeck JP: MrBayes 3: Bayesian phylogenetic inference under mixed models. Bioinformatics 2003, 19:1572-1574.

32. Shimodaira $H$, Hasegawa M: Multiple comparisons of log-likelihoods with applications to phylogenetic inference. Mol Biol Evol 1999, 16:1114-1116.

33. Swofford DL: PAUP*: Phylogenetic Analysis Using Parsimony. 2000, Version 4.0b. Sinauer Associates.

34. Whipple CJ, Zanis MJ, Kellogg EA, Schmidt RJ: Conservation of B class gene expression in the second whorl of a basal grass and outgroups links the origin of lodicules and petals. Proc Nat Acad Sci USA 2007, 104:1081-1086.

35. Sajo M, Longhi-Wagner $H$, Rudall P: Reproductive morphology of the early-divergent grass Streptochaeta and its bearing on the homologies of the grass spikelet. Plant Syst Evol 2008, 275:245-255.

36. Preston JC, Christensen A, Malcomber ST, Kellogg EA: MADS-Box gene expression and implications for developmental origins of the grass spikelet. Am J Bot 2009, 96:1419-1429.

37. Yang Z: PAML 4: phylogenetic analysis by maximum likelihood. Mol Biol Evol 2007, 24:1586-1591.

38. Yang Z, Nielsen R: Codon-substitution models for detecting molecular adaptation at individual sites along specific lineages. Mol Biol Evol 2002, 19:908-917.

39. Wong WS, Yang Z, Goldman N, Nielsen R: Accuracy and power of statistical methods for detecting adaptive evolution in protein coding sequences and for identifying positively selected sites. Genetics 2004, 168:1041-1051

40. Yang Z, Wong WS, Nielsen R: Bayes empirical bayes inference of amino acid sites under positive selection. Mol Biol Evol 2005, 22:1107-1118.

41. Vandenbussche $M$, Theissen $G$, Van de Peer $Y$, Gerats T: Structural diversification and neo-functionalization during floral MADS-box gene evolution by C-terminal frameshift mutations. Nucleic Acids Res 2003, 31:4401-4409

42. Yu H, Goh CJ: Identification and characterization of three orchid MADS box genes of the AP1/AGL9 subfamily during floral transition. Plant Physiol 2000, 123:1325-1336. 
43. Cacharrón J: Untersuchung von MADS-box genen mit der Methode der in situ-Hybridisierung in Zea mays. Ph.D. Thesis Philipps-Universität, Faculty of Biology; 1994.

44. Schmitz J, Franzen R, Ngyuen TH, Garcia-Maroto F, Pozzi C, Salamini F, Rohde W: Cloning, mapping and expression analysis of barley MADS-box genes. Plant Mol Biol 2000, 42:899-913.

45. Chang YY, Chiu YF, Wu JW, Yang CH: Four orchid (Oncidium Gower Ramsey) AP1/AGL9-like MADS box genes show novel expression patterns and cause different effects on floral transition and formation in Arabidopsis thaliana. Plant Cell Physiol 2009, 50:1425-1438.

46. Tzeng TY, Hsiao CC, Chi PJ, Yang CH: Two lily SEPALLATA-like genes cause different effects on floral formation and floral transition in Arabidopsis. Plant Physiol 2003, 133:1091-1101.

47. Huang H, Tudor M, Weiss CA, Hu Y, Ma H: The Arabidopsis MADS-box gene $A G L 3$ is widely expressed and encodes a sequence-specific DNAbinding protein. Plant Mol Biol 1995, 28:549-567.

48. Ma H, Yanofsky MF, Meyerowitz EM: AGL1-AGL6, an Arabidopsis gene family with similarity to floral homeotic and transcription factor genes. Genes Dev 1991, 5:484-495.

49. Reinheimer R, Kellogg EA: Evolution of AGL6-like MADS-box genes in grasses (Poaceae): Ovule expression is ancient and palea expression is new. Plant Cell 2009, 21:2591-2605.

50. Preston JC, Kellogg EA: Reconstructing the evolutionary history of paralogous APETALA1/FRUITFULL-like genes in grasses (Poaceae). Genetics 2006, 174:421-437.

51. Lim J, Moon YH, An G, Jang SK: Two rice MADS domain proteins interact with OsMADS1. Plant Mol Biol 2000, 44:513-527.

52. Alves SC, Worland B, Thole V, Snape JW, Bevan MW, Vain P: A protocol for Agrobacterium-mediated transformation of Brachypodium distachyon community standard line Bd21. Nature Protoc 2009, 4:638-649.

53. Mur LA, Allainguillaume J, Catalan P, Hasterok R, Jenkins G, Lesniewska K, Thomas I, Vogel J: Exploiting the Brachypodium tool box in cereal and grass research. New Phytol 2011, 191:334-347.

54. Opanowicz M, Vain P, Draper J, Parker D, Doonan JH: Brachypodium distachyon: making hay with a wild grass. Trends Plant Sci 2008, 13:172-177.

55. Brutnell TP, Wang L, Swartwood K, Goldschmidt A, Jackson D, Zhu X-G, Kellogg E, Van Eck J: Setaria viridis: A model for C4 photosynthesis. Plant Cell 2010, 22:2537-2544.

doi:10.1186/2041-9139-3-4

Cite this article as: Christensen and Malcomber: Duplication and diversification of the LEAFY HULL STERILE1 and Oryza sativa MADS5 SEPALLATA lineages in graminoid Poales. EvoDevo 2012 3:4.

\section{Submit your next manuscript to BioMed Central and take full advantage of:}

- Convenient online submission

- Thorough peer review

- No space constraints or color figure charges

- Immediate publication on acceptance

- Inclusion in PubMed, CAS, Scopus and Google Scholar

- Research which is freely available for redistribution

Submit your manuscript at www.biomedcentral.com/submit
Biomed Central 